\title{
Role of CT and MRI in Abdominal Tuberculosis
}

\author{
Anagha R. Joshi • Ashish S. Basantani • \\ Tapan C. Patel
}

Published online: 10 September 2014

(C) Springer Science+Business Media New York 2014

\begin{abstract}
Abdominal tuberculosis is a grave infection resulting in high morbidity and mortality if left untreated. The diagnostic approach to this disease has drastically changed since arrival of modern CT scan. Role of MRI for the diagnosis is yet to be fully established and is in early stages. In future, patient profile, diagnostic information required, merits and demerits of both the technologies will determine their utility. The purpose of this article is to review $\mathrm{CT}$ and $\mathrm{MR}$ imaging findings in abdominal tuberculosis.
\end{abstract}

Keywords CT $\cdot$ MRI $\cdot$ Tuberculosis

\section{Introduction}

Tuberculosis has high prevalence in densely populated regions predominantly in developing countries. Immigration and HIV prevalence have led to its persistence in developed countries. Factors which have led to its spread and persistence are myriad in nature some of them which include

This article is part of Topical Collection on Abdominal CT Imaging.

A. R. Joshi $(\bowtie)$. T. C. Patel

LTMMC \& LTMGH, Sion, Mumbai, India

e-mail: anaghajoshi2405@gmail.com

T. C. Patel

e-mail: maildr.tapan@gmail.com

A. S. Basantani

Rajawadi Hospital, Ghatkopar, Mumbai, India

e-mail: ashishofgs@gmail.com (a) Age In our country $80 \%$ of $\mathrm{TB}$ cases are seen in 15-54 age group [1]. While two-thirds of cases are males, among females, TB is disproportionately seen more in young with $50 \%$ of cases seen before the age 34 years [2].

(b) Sex TB is more prevalent in males than females. However, abdominal TB cases show female predominance $[3,4]$ in some studies, whereas others suggest equal incidence [3, 4].

(c) Concomitant medical conditions HIV is the most important risk factor for developing active tuberculosis. The risk is estimated to be between 21 and 34 times greater in people living with HIV than among those without HIV infection [5].

Other disease states that increase the risk of developing tuberculosis are diabetes mellitus, Hodgkin's lymphoma, end-stage renal disease, chronic lung disease, malnutrition, and alcoholism [6], vitamin D deficiency, etc. [6-8]. Prolonged corticosteroid therapy and other immunosuppressant drugs also increase the risk.

\section{Computed Tomography (CT Scan)}

The introduction of spiral (helical) CT in the late 1980s revolutionized CT imaging of the gastrointestinal tract by allowing faster scanning, thinner collimation $(3-5 \mathrm{~mm})$ and faster reconstruction times, as well as volume acquisition. For the first time, CT did not consist of merely a series of slices, but as a complete volume of information. This paradigm shift led to the introduction of 3D imaging with images of equal spatial resolution to be obtained in any plane which greatly expanded the clinical role of CT. It was at this time in which CT began to play a more serious 
role in evaluation of gastrointestinal tract pathology, although barium studies were still the first line of imaging for most indications.

Today, the availability of MDCT along with advancements in 3D CT imaging systems has greatly expanded the role of CT in evaluation of suspected bowel pathology. CT is now considered a first-line modality for the evaluation of a wide variety of bowel diseases.

In contrast to barium studies, CT offers the unique ability to evaluate extraluminal, peritoneal, nodal, and visceral involvement in a single examination which proves to be a major benefit in diagnosing abdominal tuberculosis.

\section{Magnetic Resonance Imaging}

MRI of abdomen has been primarily used for evaluation of solid organs like liver, spleen, pancreas, kidney, and adrenal glands because of its superior soft tissue resolution and multiplanar acquisition. However, its role in evaluation for bowel pathologies has been limited to assessment of ano-rectal abnormalities. Severe artefacts related to intestinal peristalsis, breathing and long acquisition time caused degradation of images, thus impeding the evaluation of intestinal pathologies. New advances, such as respiratory compensation, breath hold acquisition, fat suppression, and orally administered contrast agents allow us to obtain high quality MR images through the entire abdomen. Recently, some studies have investigated the value of MRI for the evaluation of small and large intestines, and this modality has become increasingly important in the diagnosis, assessment, and exclusion of small and large bowel diseases.

MRI studies of small bowel have been primarily done to evaluate inflammatory bowel diseases like Crohn's disease. MRI provides good anatomic and functional evaluation of bowel loops without any exposure to ionizing radiation.

Although, MRI study is routinely done to evaluate tuberculosis of spine, brain, and extremity joints, very few studies have been done to evaluate its role in abdominal tuberculosis. Also, the imaging findings of gastrointestinal tuberculosis using barium enema, ultrasound, and CT have been well described [4, 5]. However, very few previous reports have focused on the MR imaging findings of tuberculosis of the gastrointestinal tract.

\section{Abdominal Tuberculosis}

Abdominal tuberculosis denotes involvement of the gastrointestinal tract, peritoneum, lymph nodes, and solid viscera. It is the sixth common extra-pulmonary form of
TB after lymphatic, genitourinary, bone and joint, miliary and meningeal tuberculosis [9, 10].

Infection by Mycobacterium tuberculosis causing abdominal tuberculosis is acquired in following ways:

(1) Dissemination of primary pulmonary tuberculosis in childhood.

(2) Swallowing of infected sputum in active pulmonary tuberculosis.

(3) Hematogenous dissemination from a focus of active pulmonary tuberculosis or miliary tuberculosis.

(4) Mycobacteria can spread from infected adjacent organs like fallopian tubes.

(5) Intestinal infection can occur by lymphatic spread from infected mesenteric lymph nodes.

(6) Mycobacteria can also get disseminated through bile from tubercular granulomas of the liver.

Tuberculous Lymphadenitis

Tuberculous lymphadenopathy is the most common manifestation of abdominal tuberculosis and occurs in 25-93\% of cases [11]. The mesenteric, omental, peripancreatic and upper paraaortic lymph nodes are frequently involved which is explained by lymphatic drainage from the main areas of infection: small bowel, ileocecum, right side of the colon, liver and spleen. Lower paraaortic lymph nodes involvement is very rare and is seen in systemic hematogenous spread or from direct spread from the reproductive organs.

Pathologically tuberculous infection of a lymph node follows a spectrum from early exudative to caseous to late fibro-calcification phases.

\section{$C T$}

Four types of contrast patterns have been described by Pombo et al. [12] on contrast-enhanced CT in patients of abdominal tuberculosis:

(a) peripheral rim enhancement with hypodense centers,

(b) inhomogeneous enhancement,

(c) homogeneous enhancement and

(d) nonenhancing nodes

They concluded that, neither the nodal attenuation values nor the patterns of enhancement are characteristic of tuberculosis; however, adenopathy showing peripheral rim-enhancement with relative low attenuation centers can suggest a diagnosis of tuberculosis in appropriate clinical settings.

Lymph nodes showing peripheral enhancement with hypodense centers, secondary to caseous and/or 
Fig. 1 Pattern of lymph node enhancement on CT: a Enlarged homogeneously enhancing mesenteric lymph nodes; b Enlarged heterogeneously enhancing retroperitoneal lymph nodes; c Peripheral rim enhancing mesenteric lymph nodes; d Conglomerate mesenteric lymph node mass; e Cluster of normal sized mesenteric lymph nodes; f Calcific focus in aortocaval lymph node
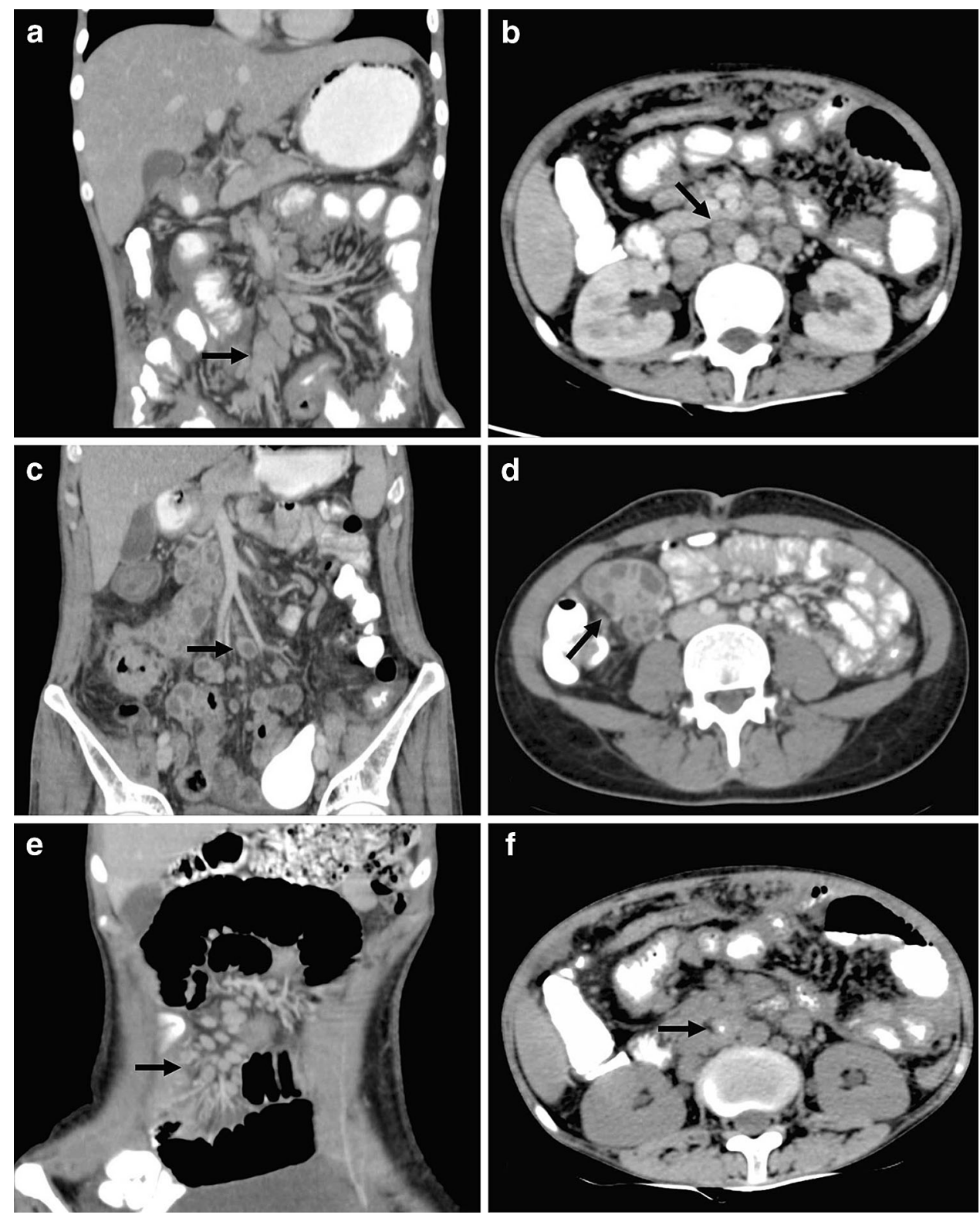

liquefactive necrosis were the most common group. Other patterns [13] include:

(e) Conglomerate lymph node masses with areas of necrosis secondary to perinodal inflammation.

(f) Increased number ( $>3$ in one CT section) of normal sized or mildly enlarged mesenteric nodes of homogeneous density.

(g) Calcified lymph nodes (Fig. 1a-f)

Lymph nodes with hypodense centers are also seen in metastatic malignancy, lymphoma after treatment, inflammatory conditions (such as Crohn's disease), pyogenic infection and Whipple's disease, however, other imaging findings and clinical features are helpful in diagnosis [13].
Similarly, calcified lymph nodes are also seen in metastases from teratomatous testicular tumors and nonHodgkins lymphoma after treatment [14]. However, nodal calcification in patients from endemic areas in the absence of known primary malignancy suggests a tuberculous etiology [15].

\section{MRI}

In 2005 De Backer et al. [16•] studied MRI features of abdominal lymphadenopathy in 13 patients.

MR signal intensities of 41 lymph nodes studied were as follows 
Fig. 2 Lymph node pathology on MRI a Cor T2 fat suppressed: conglomerate mesenteric lymph node mass showing heterogeneous hyperintense $\mathrm{T} 2$ signal with hypointense rim and high signal area (arrowhead) b Cor contrast-enhanced $\mathrm{T} 1$ fat suppressed: Conglomerate lymph node mass same as in (a), Showing peripheral and central enhancement. c Ax T2 fat suppressed: Enlarged lymph node showing homogeneous hyperintense signal. d Ax contrast-enhanced T1 fat suppressed: Same lymph node as in (a) showing homogeneous enhancement. e Ax T2 fat suppressed: Enlarged lymph nodes showing heterogeneous hyperintense signal. f Ax contrast-enhanced $\mathrm{T} 1$ fat suppressed: Heterogeneous enhancement of the lymph nodes seen in (e)
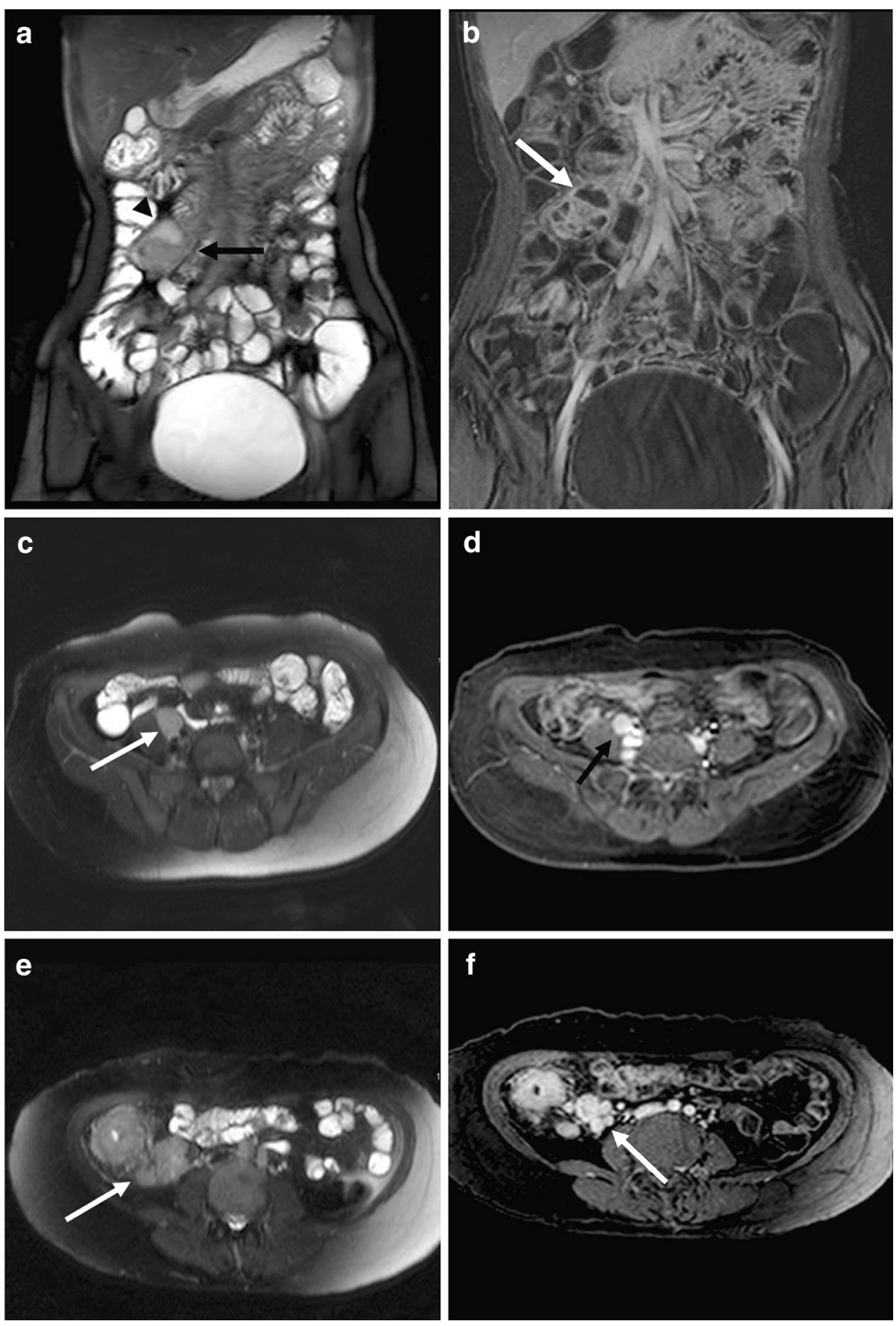

(1) All lymph nodes ( $n=40,97.6 \%)$ except 1 , were hyperintense on T2. Perinodal T2-hyperintensity was present in $23(56.1 \%)$ lymph nodes. Similar to the T2-weighted appearance of an intracranial tuberculoma, the signal intensity may differ depending on the stage of evolution, with central hyperintensity on T2weighted images corresponding to liquefactive necrosis, and central hypointensity resulting from the presence of paramagnetic free radicals secreted from active phagocytic cells. Perinodal hyperintense signal is suggestive of capsular disruption.

(2) On T1-weighted images, 30 (73.2\%) lesions were hypointense and $11(26.8 \%)$ isointense.

(3) Post contrast T1-weighted images show different enhancing patterns. The enhancement patterns include peripheral enhancement in 28 (68.3\%) 
patients visible as uniform, thin or thick, complete or incomplete rim like, and a conglomerate group of nodes showing peripheral and central areas of enhancement. Heterogeneous and homogeneous enhancements were present in $10(24.4 \%)$ and 3 (7.3\%) lymph nodes, respectively.

In our experience, lymph nodes were involved in $90 \%$ patients and peripheral and heterogeneously enhancing lymph node groups showed equal incidence. On MRI lymph nodes showed predominantly heterogeneous hyperintense signal with some of them showing perinodal hyperintnesity suggestive of capsular disruption on $\mathrm{T} 2 \mathrm{~W}$ sequences (Fig. 2). On T1 W sequence, hypointense lymph nodes showed peripheral enhancement and isointense lymph nodes showed homogeneous enhancement. (Fig. 2a-d)

Comparison of lymph nodes having necrosis was made between MRI and CT. The largest lymph node showing areas of necrosis was compared on plain, contrast CT and plain, contrast MRI. Lymph nodes showing peripheral enhancement with central nonenhancing necrotic area were included for comparison. Findings are as follows:

\begin{tabular}{llllll}
\hline & \multicolumn{2}{l}{$\begin{array}{l}\text { Computed } \\
\text { tomography }(\mathrm{CT})\end{array}$} & & \multicolumn{2}{l}{$\begin{array}{l}\text { Magnetic resonance imaging } \\
\text { (MRI) }\end{array}$} \\
\cline { 2 - 3 } \cline { 5 - 6 } & $\begin{array}{l}\text { Plain } \\
\mathrm{CT}\end{array}$ & $\begin{array}{l}\text { Contrast- } \\
\text { enhanced } \\
\mathrm{CT}\end{array}$ & & $\begin{array}{l}\text { Plain } \\
\text { MRI (T2 } \\
\text { weighted) }\end{array}$ & $\begin{array}{l}\text { Contrast-enhanced } \\
\text { MRI (Post contrast } \\
\text { T1 fat suppressed) }\end{array}$ \\
\hline $\begin{array}{l}\text { No. of } \\
\text { patients }\end{array}$ & 3 & 12 & $9(75 \%)$ & $12(100 \%)$ \\
\hline
\end{tabular}

Thus, in our experience plain MRI is more sensitive than plain CT, also lymph nodes with post contrast enhancement are better visualized on MRI compared to CT due to better soft tissue resolution.

\section{Gastrointestinal TB}

The gastrointestinal tract is involved in $65-78 \%$ of the abdominal TB cases [17].

Tuberculosis can involve any part of the gastrointestinal tract from mouth to anus, the peritoneum and the pancreatico-biliary system. Clinical presentation is often confusing with other common and rare diseases due to nonspecific symptoms.

Disease within any part of the GI tract itself has two main forms: [1] an ulcerative process and [2] a hyperplastic reaction.

By far, the commonest site of involvement in the bowel is the terminal ileum and the ileocecal region, as shown by the high incidence of involvement of these regions in the different series [18-21]; possibly because of the increased physiological stasis, increased rate of fluid and electrolyte absorption, minimal digestive activity and an abundance of lymphoid tissue at this site.

\section{$C T$}

The most common CT finding is mural thickening affecting the ileocecal region, either limited to the terminal ileum or cecum or, more commonly, involving both regions simultaneously, associated with pericecal fat stranding. The degree of bowel wall thickening may vary from slight at early stage to severe resulting in acute obstruction at later stages (Fig. 3). In more advanced disease, gross wall thickening, adherent loops, large regional nodes, and mesenteric thickening can together form a soft tissue mass centered around the ileocecal junction. Also fibrosis may result in pulled up cecum. In some cases, hypodense areas most likely to represent necrosis may be noted within the thickened wall [22].

Skip areas of concentric mural thickening may be seen in small bowel loops, usually affecting the ileal loops (Fig. 4). These segments may also show luminal narrowing, with or without proximal dilatation. In our experience, oral contrast is helpful to gage the extent of bowel thickness and mucosal irregularity in these cases provided with acute bowel obstruction which is ruled out. However, mucosal enhancement pattern recognition is futile in cases with oral contrast. T1 W-MRI may help in mucosal enhancement recognition due to superior contrast between enhancing bowel wall and lumen.

\section{MRI}

In 2006 De Backer et al. [23•], studied features of MRI in gastrointestinal tuberculosis with $\mathrm{CT}$ correlation in 6 patients.

According to this study on MRI, abnormal bowel wall showed decreased signal intensity compared to normal bowel wall on unenhanced T1-weighted images and slightly increased, and slight heterogeneous signal intensities on T2-weighted images. Symmetric and asymmetric thickening of cecum were visualized. T2-weighted imaging is critical for characterizing the cause of wall thickening because an area of high signal intensity is seen in the presence of active inflammation, whereas fibrostenotic disease is accompanied by areas of normal or lower signal intensity [24].

T2-weighted sequences are very sensitive for detecting inflammation of the bowel loop more so with negative oral contrast agents like superparamagnetic iron oxide particles; and fat-suppressed sequences which suppress the 
Fig. 3 Ileocecal tuberculosis a Cor contrast-enhanced CT: mildly enhancing circumferential symmetrically thickened cecal wall. b Cor T2 fat suppressed: Cecal wall thickening showing heterogeneous hyperintense signal. c, d Cor Pre-contrast T1 weighted and contrast-enhanced T1 fat suppressed: Thickened cecal wall showing isointense signal, which shows post contrast enhancement in same patient as in (a). The enhancement on MRI has much better visibility compared to CT
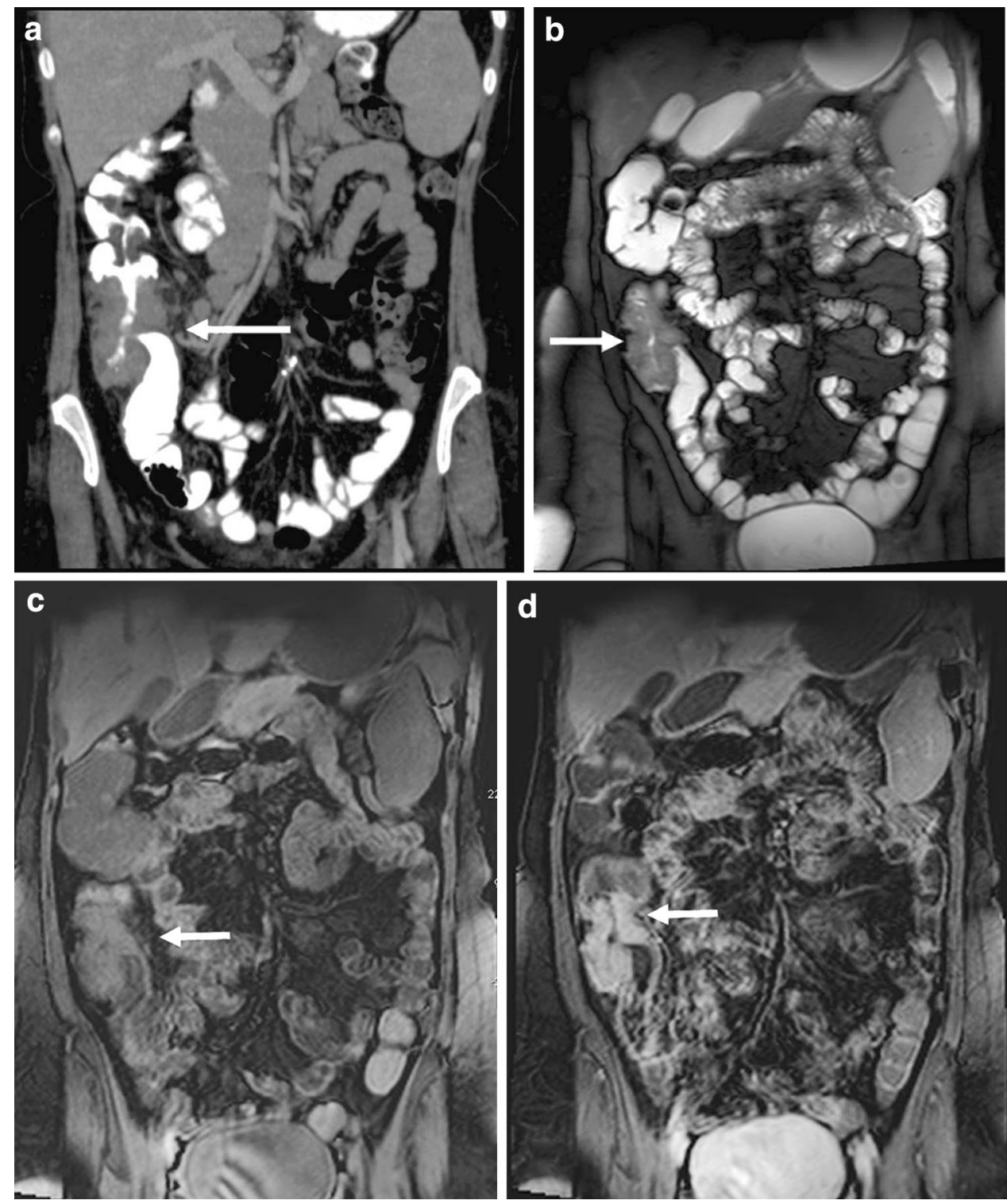

mesenteric fat signal. Also fat-suppressed T2-weighted imaging helps us to observe $\mathrm{T} 2$ hyperintense signal extrinsic to inflamed bowel wall due to local mesenteritis.

ln our experience, oral contrast $(2.5 \%$ mannitol or Volumen) is essential for adequate bowel distension in MRI. Also prone position helps to separate bowel loops and reduces breathing artefacts. The structural bowel details are well visualized on both CT and MRI, but cine sequence in MRI (Fig. 5) can help in bowel peristalsis assessment which will help to detect subtle strictures. [22]

Peritoneal Tuberculosis

Peritoneal involvement in tuberculosis occurs primarily by hematogenous spread but may be secondary to ruptured lymph nodes, a perforated gastrointestinal lesion, or fallopian tube involvement.

Peritoneal tuberculosis is traditionally divided into three types:

(1) "Wet" with free or loculated ascites with strands of fine adhesions and floating membranes within fluid [25] (Figs. 6, 7).

(2) "Dry plastic" with mesenteric thickening, caseous lymph nodes and fibrous adhesions; and

(3) "Fibrotic fixed" with mass formation of omentum and matting of bowel loops with cocooning in some cases [26].

There is considerable overlap between three types on CT. 
Fig. 4 Small bowel thickening. a Coronal reconstructed plain CT: multiple small bowel segments showing mildly thickened wall. b, c Ax T2 fat suppressed: Thickened small bowel wall showing isointense signal intensity. d, e Ax contrast-enhanced $\mathrm{T} 1$ fat suppressed: Enhancing thickened small bowel wall
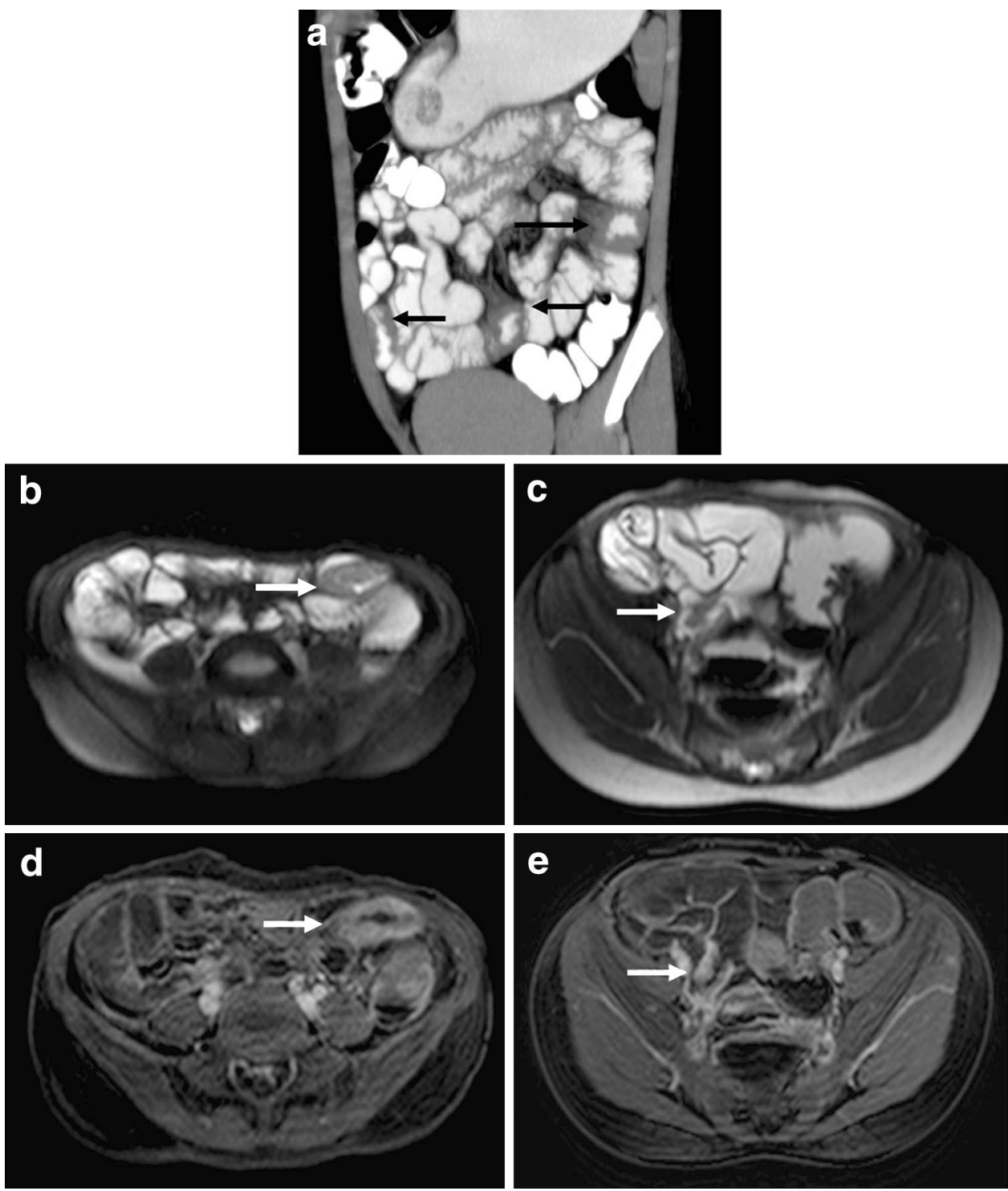

Peritoneal tuberculosis is mainly manifested on CT by varying degrees of mesenteric and/or omental infiltration with (wet type) or without (dry type) associated ascites [22].

Mesenteric changes is characterized by nodular infiltration of mesentery with both micro and macronodules seen within mesentery and along mesenteric aspect of small bowel loops. Thickening of mesentery with varying appearances based on extent of mesentery involvement such as stellate appearance, generalized increase in fat density due to infiltration, edema may be noted [27]. Few advanced cases may show soft tissue density masses in mesentery causing adherence of adjacent mesenteric leaves.

Ascites in tuberculosis is usually of high density (25-45 HU) and may be with characteristic of TB [13]. High density is explained by the high protein and cellular debris in tuberculous exudate. However, water density may also be seen secondary to immune reaction.

Peritoneal enhancement is usually associated with smooth uniform thickening of the peritoneum [26, 28] with scattered nodules noted in some cases. However, nodular implants with irregular thickening should also raise the suspicion of peritoneal carcinomatosis in appropriate clinical setting.

Omental infiltration (Fig. 7) is seen in 3 patterns i.e., smudged, omental cake (Fig. 8) and nodular, with smudged pattern being most common [28]. This pattern is characterized by diffuse increase in density of omentum, whereas omental cake appears as thickened omentum enclosing the peritoneal cavity

In our experience of MRI in these cases, thickened peritoneum showed iso to hyperintense signal on T2weighted images with post-contrast enhancement. The 

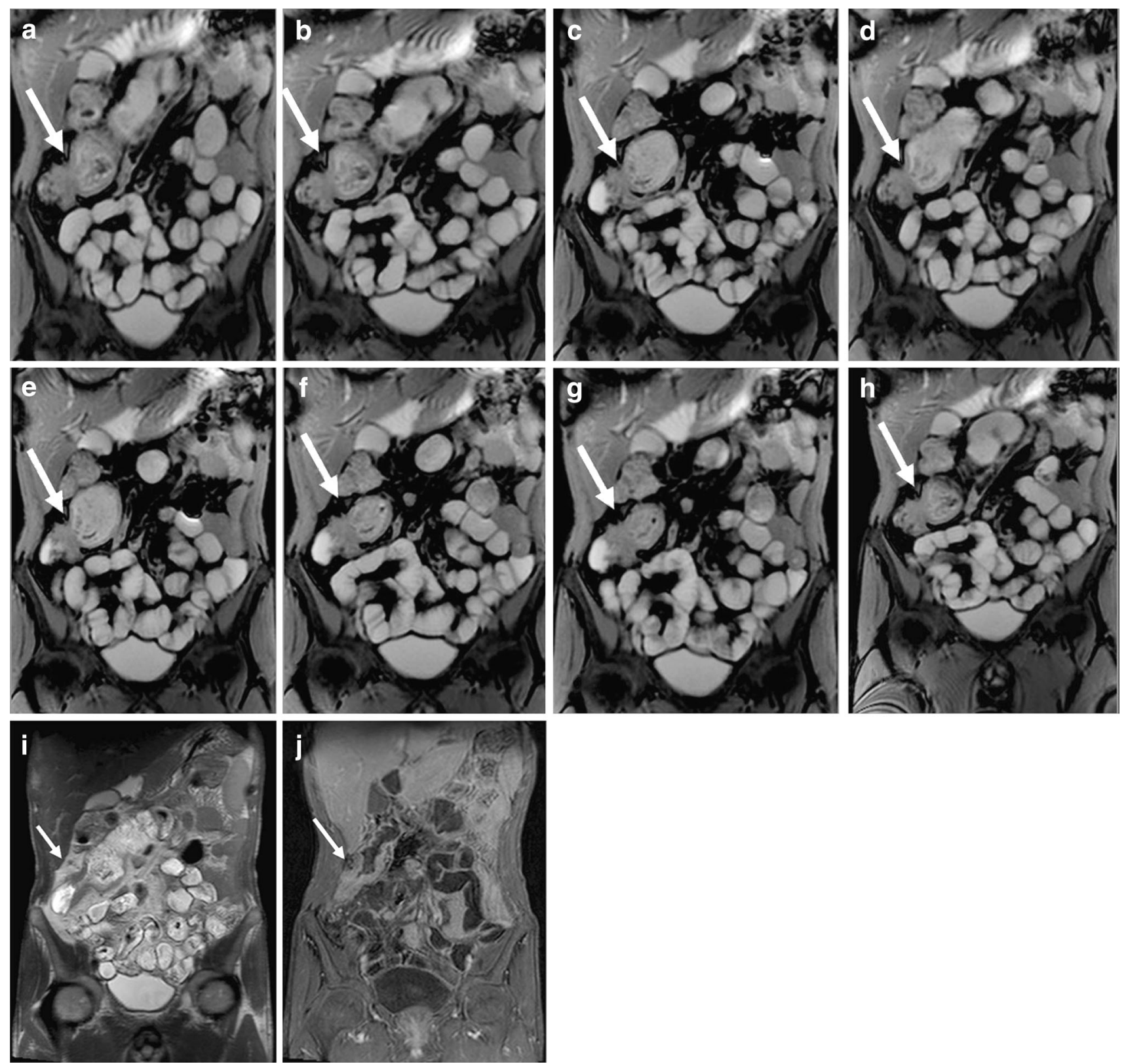

Fig. 5 Terminal ileal stricture. a-h Dynamic Balanced Turbo Field Echo (BTFE)[cine MRI]: Few of multiple sequential images, taken at the same position in real time, showing terminal ileal stricture (arrow) with proximal dilated small bowel loop showing alternate relaxation

and contraction. Ileal stricture showed no relaxation. i Cor T2 weighted: terminal ileal stricture. $\mathbf{h}$ Cor contrast-enhanced $\mathrm{T} 1$ fat suppressed: Enhancing wall of terminal ileal stricture in same patient

peritoneal and mesenteric nodules noted in CT could not be well delineated on MRI due to poor spatial resolution. All the patients showed thick and thin septae within the loculated fluid on T2-weighted sequences with mild enhancement on contrast-enhanced T1weighted sequences which were not seen either on plain or contrast-enhanced CT (Fig. 6). Thickened omentum showed heterogeneous hyperintense signal on T2weighted sequences.

Solid Viscera TB

\section{$C T$}

Hepatobiliary $T B$ Isolated hepatic tuberculosis is rare; however, miliary tuberculosis in liver is the most common form of hepatic tuberculosis and was found in $80-100 \%$ of autopsied patients with disseminated tuberculosis [29]. 
Fig. 6 Peritoneal tuberculosis. a Ax contrast-enhanced CT; b Ax T2 weighted; Smoothly thickened enhancing peritoneum. Thickened peritoneum appears hyperintense on $\mathrm{T} 2$ weighted image. c Ax contrast-enhanced CT: Loculated ascites in pelvis with enhancing peritoneum. d Ax T2 fat suppressed: Loculated ascites with multiple thin septae within ascites
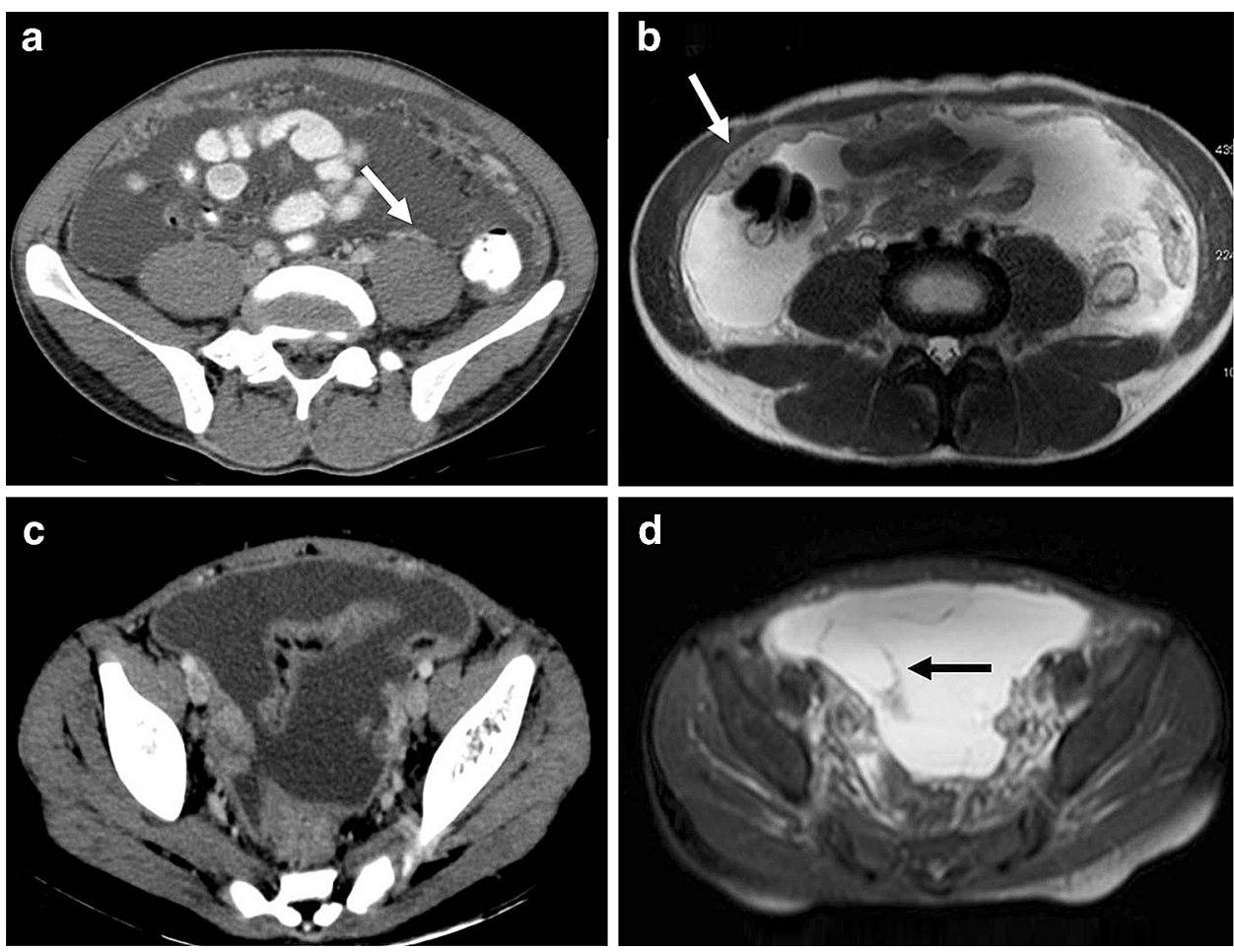

Hepatic TB occurs primarily in three type which include parenchyma type, tuberculous cholangitis, and serohepatic type, with parenchyma type further divided into 3 subtypes: miliary, nodular, and mixed [30॰].

(i) Parenchyma type

Miliary type It is depicted as hepatomegaly with multiple or diffuse, hypodense miliary micronodular lesions $(\leq 2.0 \mathrm{~cm}$ in diameter on $\mathrm{CT})$, often as a part of tuberculosis in the whole body. Few foci of miliary calcifications may also be visualized.

Nodular type This type shows lesions with a diameter $>2 \mathrm{~cm}$, is less common and has been found to be fused miliary or micronodular lesions.

The lesion may vary from being hypodense to cystic density due to caseous necrosis. It may show foci of punctate calcification within, which help in diagnosis. Mixed type Mixed type reveals findings comprising of miliary calcifications with nodular lesions. It has been suggested these findings represent different pathological stages of tuberculosis like granulomas, liquefaction necrosis, fibrosis and calcification coexisting together.

(ii) Tuberculous cholangitis

Tuberculous cholangitis is rare and occurs mainly in children. Obstructive jaundice is the most common presentation.

CT reveals irregular dilated intrahepatic ducts or diffuse miliary calcifications along the course of the bile ducts. The latter was considered as a typical feature of tuberculous cholangitis [31].

(iii) Serohepatic type

This is the rarest form and CT reveals multi-nodular hypodense lesions in the subcapsular region of liver with thickened capsule.

Gall Bladder TB Gall bladder TB is extremely rare because the gallbladder is highly resistant to tubercular infection, which is possibly due to the inhibitory function of bile [32, 33].

Gall bladder TB usually presents as cholecystitis or as a gall bladder mass. Cholelithiasis and cystic duct obstruction are considered the most important factors in the development of gallbladder TB $[32,33]$.

Splenic Tuberculosis Splenic TB occurs in two forms.

The first form, which is more common presents itself during miliary $\mathrm{TB}$, especially in immunocompromised patients. The spleen is the third most common organ infected in miliary TB (lung $100 \%$, liver $82 \%$, spleen $75 \%$, lymph nodes $55 \%$, bone marrow $41 \%$ ) [34].Grossly, spleen is enlarged and shows multiple varying sized tiny nodules scattered throughout parenchyma with some nodules coalescing with each other to form microabscesses.

Other unusual form of splenic TB is the primary involvement of the spleen. These patients are immunocompetent and 
Fig. 7 Omental infiltration. a Ax contrast-enhanced CT; b T2 fat suppressed; c Ax contrast-enhanced $\mathrm{T} 1$ fat suppressed: Thickened omentum with smudged pattern of infiltration. Omentum shows heterogeneous hyperintense signal on T2. d Ax contrastenhanced CT; e Ax T2 weighted: Omentum infiltrated with multiple nodules
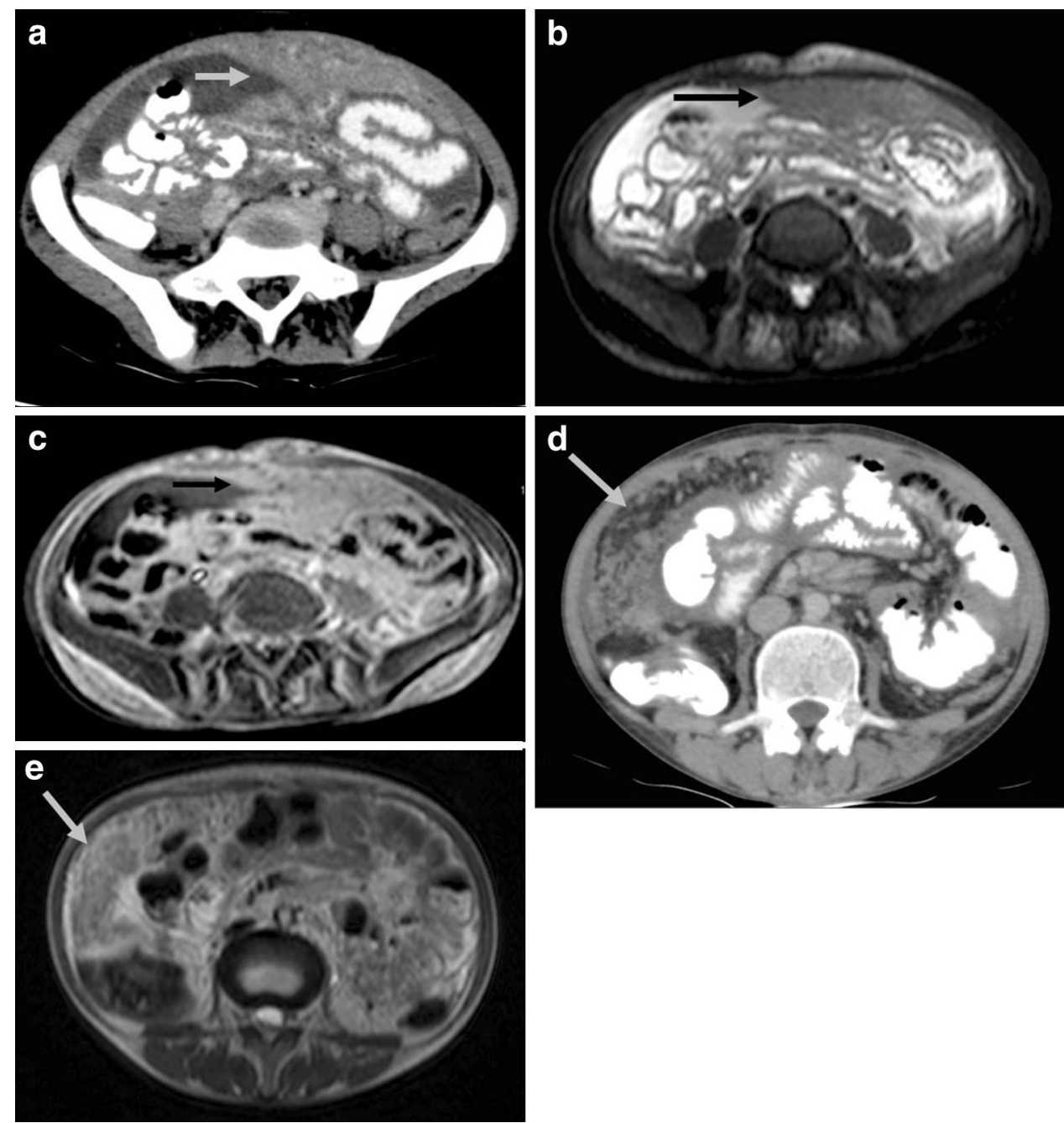

there are sporadic case reports of splenic TB in immunocompetent patients.

Splenic TB most commonly occurs in cases of miliary TB and rarely in isolated form. CT reveals splenomegaly with or without multiple hypodense nodules of varying size with few of them coalescing to form hypodense masses. These hypodense nodules have HU of 35-45.

However, the findings of miliary TB involving liver and spleen are non-specific. The differential diagnosis of hepatosplenic tuberculosis includes sarcoidosis; fungal infections such as histoplasmosis, brucellosis or berylliosis; Hodgkin's disease; parasitic diseases; and metastatic carcinoma [29].

Associated clinical and laboratory features help in diagnosis.

Pancreatic Tuberculosis Pancreatic tuberculosis is considered to be an extremely rare clinical entity.

The pancreas is biologically protected from tuberculosis, probably because of the presence of pancreatic enzymes that interfere with the seeding of Mycobacterium tuberculosis [35]. Pancreas is involved by direct extension, lympho-hematogenous dissemination, or following reactivation of previous abdominal tuberculosis [36].

CT findings of pancreatic TB are variable and vary from pancreatitis, pancreatic abscesss to pancreatic carcinoma [37-40].

In 1997, Pombo et al. [12] studied pancreatic TB in 6 (3 non AIDS and 3 AIDS) cases and reported that pancreatic mass lesion causing focal enlargement of the gland was the most common CT finding and was found in all the nonAIDS patients and in one AIDS patient with peripheral rim enhancing enlarged lymph nodes.

\section{MRI of Solid Viscera}

In 2004, Yu et al. [30•], studied CT and MR features of hepatic tuberculosis and varying imaging patterns of tuberculous lesions in liver. 
Fig. 8 Omental caking with clumped bowel loops. a Ax contrast-enhanced CT; b Ax T2 fat suppressed; c Ax contrastenhanced T1 fat suppressed: Thickened enhancing omentum forming a cake with clumping of bowel loops. d Cor contrastenhanced CT; e Cor T2 weighted: clumped bowel loops with early 'cocooning' and loculated ascites showing thin septae on MRI

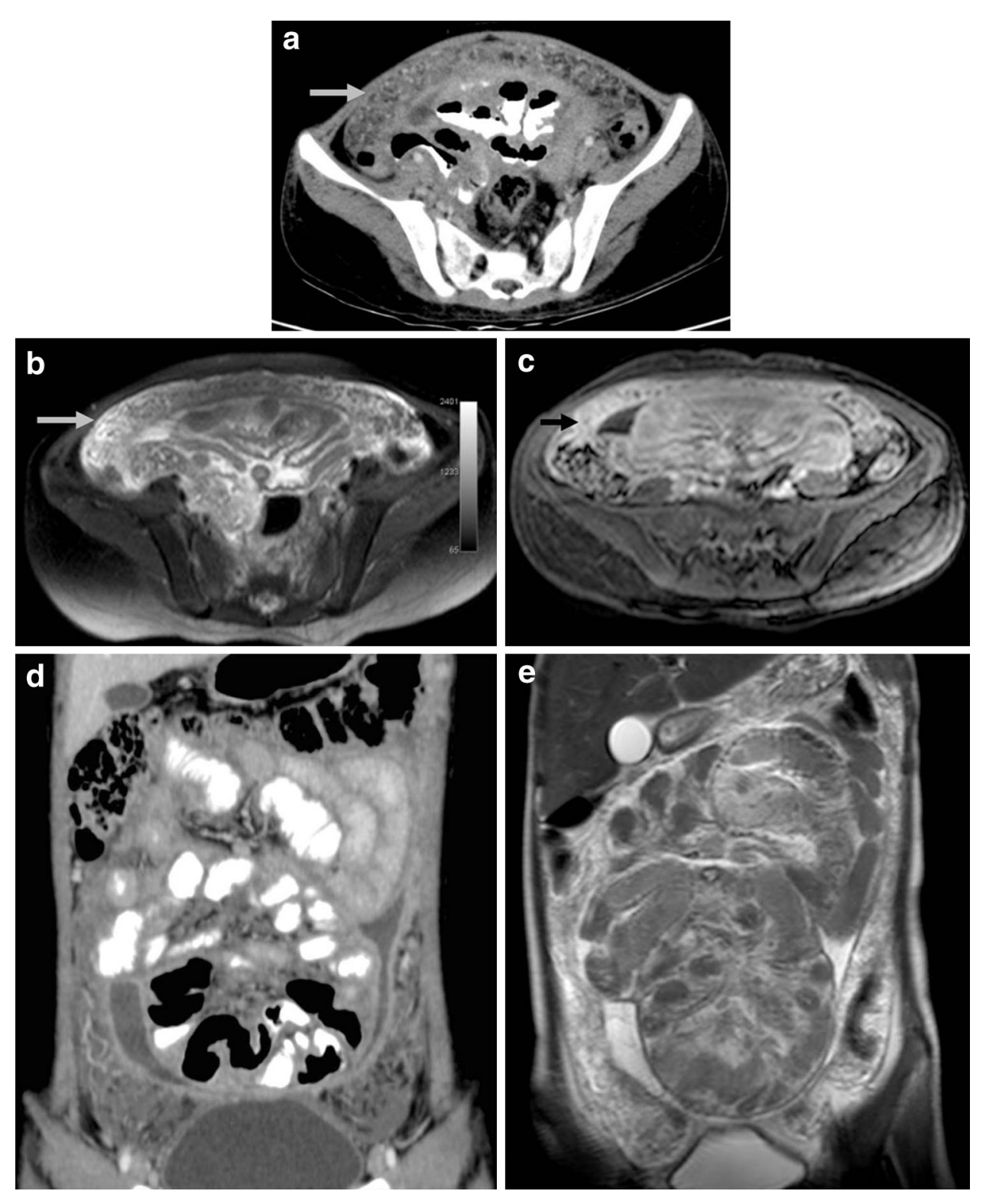

MRI of hepatic tuberculosis showed a hypointense nodule with a hypointense rim on T1-weighted imaging, and hypointense, isointense or hyperintense with a less intense rim on T2-weighted imaging, and peripheral enhancement or internal septal enhancement on post-contrast MRI. MR findings were related to different pathological stages of tuberculosis. At the early and medium stages of granuloma with or without caseation or liquefaction necrosis, the lesion showed low signal intensity on T1-weighted imaging and high signal intensity on T2weighted imaging. Similar lesions with hypointense signal on T1-weighted imaging and hypo- and isointense on T2weighted imaging were corresponding to fibrous stage of tuberculosis or could be due to free radicals released by active macrophages and may have slightly or no peripheral enhancement. Tuberculous granuloma at early or medium stage and fibro-proliferous lesions were all depicted as a low-density area on CT but as various signal intensities on T2-weighted imaging, which is the main advantage of MRI in the diagnosis of hepatic tuberculosis. But MRI is of limited value for detecting calcification. Calcific lesions on CT may appear hypointense on T2-weighted MRI images.

\section{Splenic TB}

On MRI, tuberculous focal splenic lesions may show variable signal intensities and enhancement patterns after IV administration of gadolinium. This spectrum of variable imaging findings may represent different phases of disease 
Fig. 9 Hepatosplenic tuberculosis in HIV a, b Ax contrast-enhanced CT: hypodense lesions in liver and spleen with heterogeneous enhancement in splenic lesions. c, d Ax T2 fat suppressed: Multiple lesions in liver and spleen showing hyperintense signal in liver and heterogeneous signal with hyperintense rim in spleen in same patient. e, f Ax contrastenhanced T1 fat suppressed: Multiple peripherally enhancing lesions seen in both liver and spleen, with splenic lesions showing heterogeneous enhancement. MRI demonstrates more number of lesions with better enhancement pattern than $\mathrm{CT}$
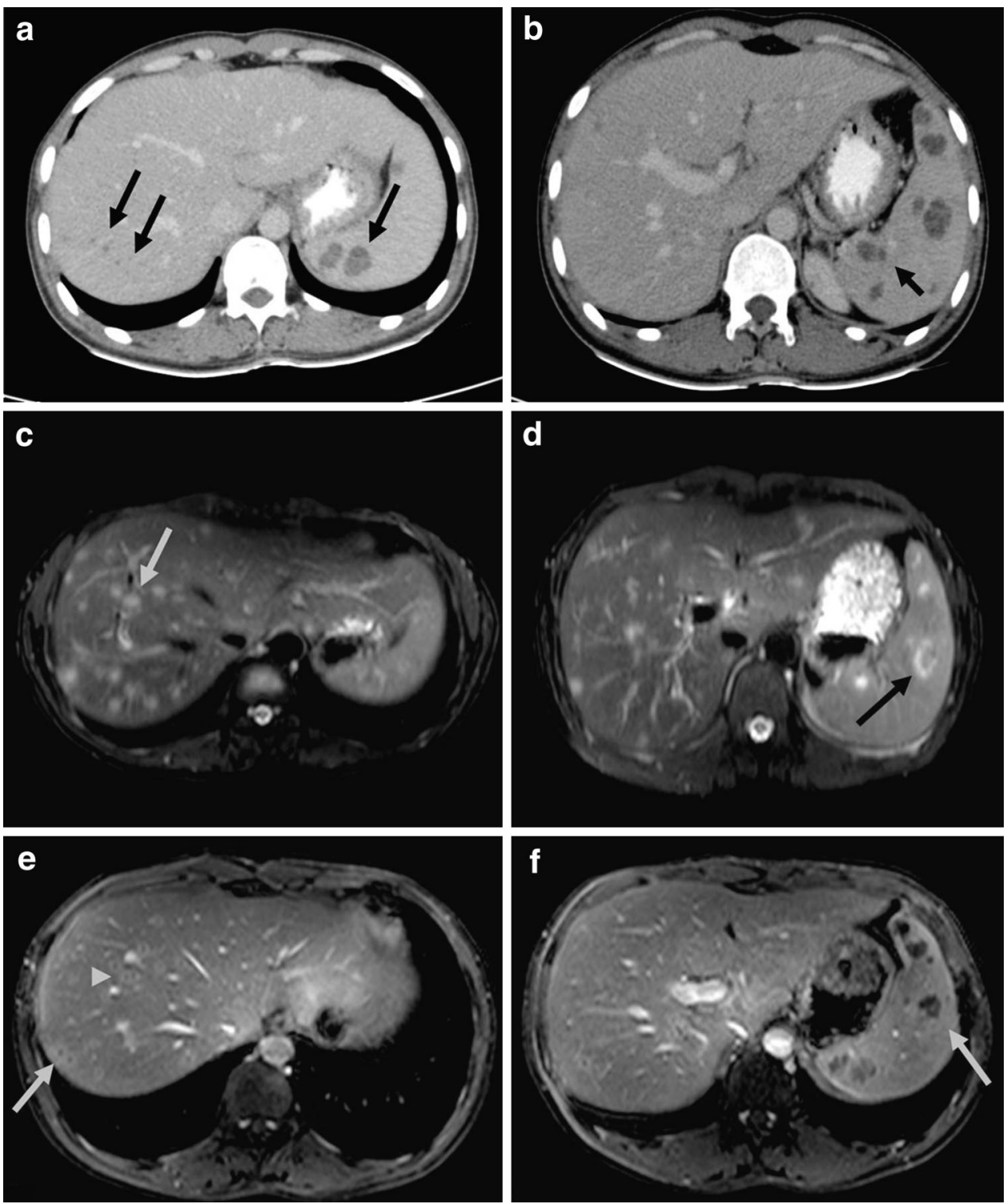

progression corresponding to different degrees of fibrosis, granuloma formation, caseation, and liquefaction necrosis [41]. Lesion hypointensity on T2-weighted images is thought to be due to the presence of free radicals produced by macrophages during active phagocytosis, may be associated with increased fibrosis and granulomatous tissue, or may reflect the presence of calcifications [42], [43•]. The finding of lesion hypointensity on T2-weighted images may be a helpful characteristic for differentiating splenic tuberculoma from other neoplastic or inflammatory lesions. Furthermore, a hypointense nodule with a less hypointense rim on T1- weighted images and a hyperintense central area with a less intense rim on T2-weighted images have also been reported. These findings reflect histologic differences between the center and the periphery of the tuberculous lesion [44]. These findings may reflect caseating granuloma with a liquid center and peripheral reactive fibrosis. Finally, a hyperintense mass without rim hypointensity on T2-weighted images may also be noted. The latter finding may reflect extensive central liquefaction necrosis with only minimal peripheral granuloma formation or fibrosis (or both).

In our experience sensitivity for detecting granulomas was higher on MRI than on CT. Also the visualized lesions showed better contrast (Fig. 9).

\section{Adrenal tuberculosis}

Adrenal tuberculosis is the one of the common causes of adreno-cortical insufficiency (Addison's disease). It is usually bilateral and occurs together with the presence of extra-adrenal tuberculosis, such as pulmonary, gastrointestinal or peritoneal tuberculosis (Fig. 10). Calcifications on CT are the most important diagnostic clue. In adrenal 
Fig. 10 Adrenal tuberculosis. a, b Ax and Cor contrastenhanced CT: enlarged right adrenal gland with preserved contours. Bilateral adrenal glands show hypodense peripherally enhancing lesions. c Ax T2 fat suppressed: Right adrenal lesion noted in (a) shows hypointense signal with peripheral hyperintense rim. d Cor contrast-enhanced T1 fat suppressed: Right adrenal hypointense lesion with peripheral enhancement
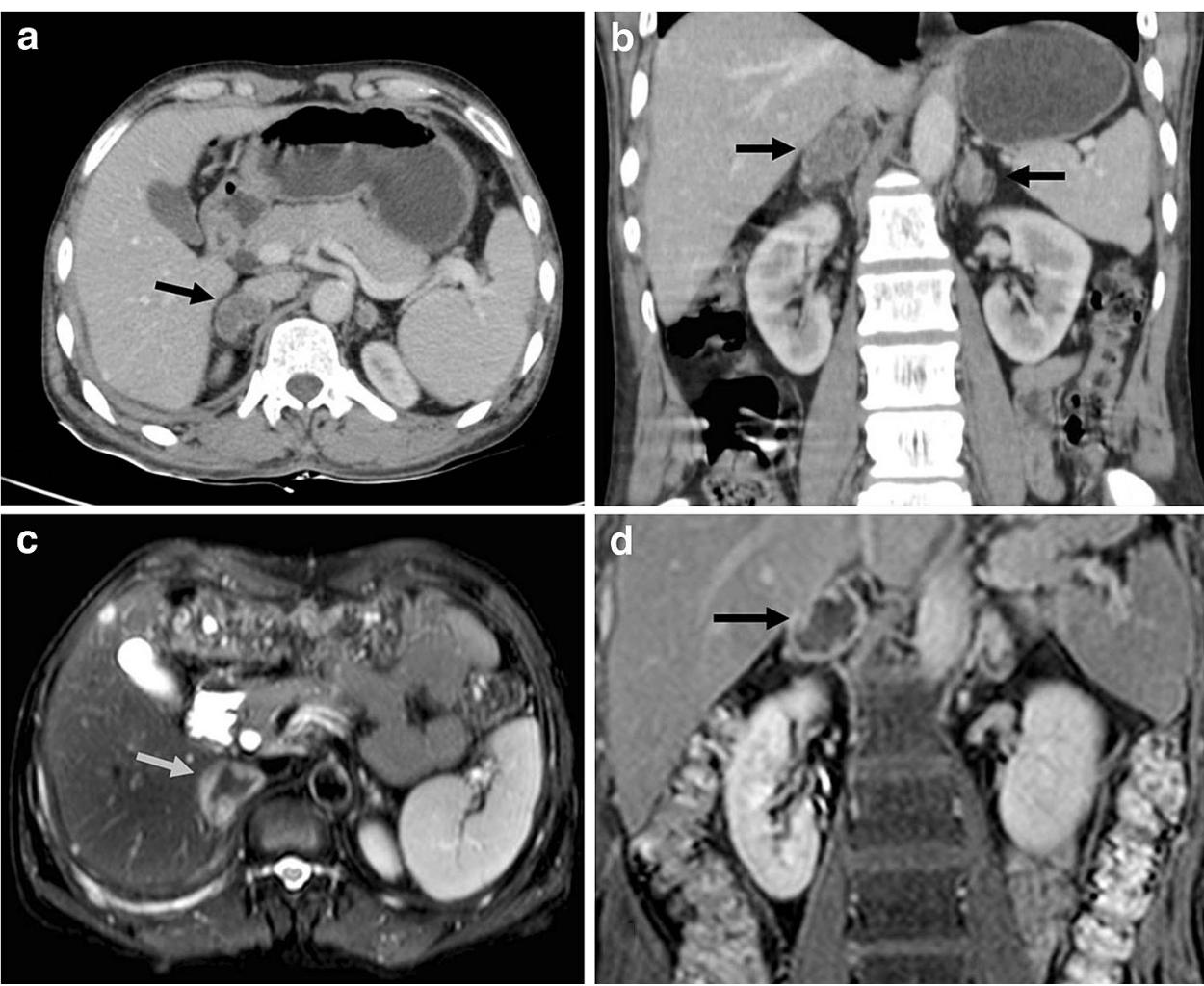

TB with masses in the gland, the contour of the gland is preserved. Adrenal TB should be suspected in patient with known tuberculosis and clinical presentation of Addison's disease.

In a study of 34 patients with adrenal tuberculosis by Yang et al. [28], bilateral adrenal gland involvement was seen in $91 \%$ patients. Other important features were calcification, peripheral enhancement, and preserved contour of the adrenal glands.

On MRI, signal intensity of adrenal TB can vary depending on the pathologic stage of the disease. In a study of MRI in 18 patients of adrenal tuberculosis by Zhang et al. [45•], adrenal glands were bilaterally involved in $89 \%$ patients. The central region of the enlarged glands demonstrated $\mathrm{T} 2$ hypointense or isointense signal intensity in $21(62 \%)$ and T2 hyperintense in $12(38 \%)$. Among the 18 enlarged glands that had contrast MR, $15(83 \%)$ had peripheral rim enhancement, $1(6 \%)$ had homogeneous enhancement, and the last two $(11 \%)$ had heterogeneous enhancement.

\section{Clinical Features}

Abdominal tuberculosis may present at any age but commonly seen in young adults. The spectrum of disease in children is different from adults, in whom adhesive peritoneal and lymph nodal involvements are more common than gastrointestinal disease [46].

Age and Sex distribution Two-thirds of the patients are 21-40 year old.

There are conflicting reports regarding the male v/s female predominance. Some studies suggest that incidence is equal whereas others show female predominance [3, 4, 47-51]. In our experience female prevalence was much higher.

Abdominal TB is one of few diseases that can affect any abdominal organ. The modes of presentation may vary from acute to acute-on-chronic to chronic symptomatic disease or it may be an incidental finding at laparotomy for other diseases. The clinical manifestations depend on the site and type of involvement. The symptomatology mainly includes (i) constitutional symptoms in about one-third of patients and (ii) local symptoms and signs referable to the site involved.

The common clinical symptoms are

(A) Constitutional symptoms which are seen in $1 / 3$ of the cases [17] include fever (low grade with evening rise of the temperature), weight loss, anorexia, generalized weakness, and night sweats.

These symptoms are frequently seen in those with ulcerative intestinal lesions and ascitic peritoneal tuberculosis [52]. 
(B) Local symptoms

(1) Abdominal pain is the most common symptom. Pain can be either colicky due to luminal compromise, or dull and continuous when the mesenteric lymph nodes are involved.

(2) Symptoms of intestinal obstruction which include vomiting, constipation, distension of abdomen.

(3) Distension of abdomen in patients with ascites.

(4) Diarrhea, in cases with ulcerative lesions which may lead to malabsorption.

(5) Altered bowel habits with alternating diarrhea and constipation.

(6) Hematemesis in gastroduodenal lesions occurs occasionally.

(7) Hematochezia in colonic lesions.

(8) Jaundice as a very rare presentation in patients with hepatic, pancreatic and periportal lymph node involvement.

(9) Addison's disease in cases of adrenal tuberculosis characterized by dizziness due to persistent hypotension, weakness, fatigue, weight loss.

\section{Diagnosis}

The isolation of acid fast bacilli (AFB) is the gold standard for diagnosis of pulmonary tuberculosis but may not be possible for establishing the diagnosis of various forms of abdominal tuberculosis. So far, the diagnosis of abdominal tuberculosis has been made either on the histological evidence of TB in the tissue (e.g., evidence of tubercles with caseation or demonstration of AFB in a lesion) or typical operative findings suggestive of TB or animal inoculation or tissue culture yielding the growth of M. tuberculosis. Now, with the advent of better radio-imaging procedures, new criteria for the diagnosis suggested by Lingenfelser [53] were as follows:

i. Clinical manifestations suggestive of TB

ii. Imaging evidence indicative of abdominal TB

iii. Histopathological or microbiological evidence of TB and/or

iv. Therapeutic response to treatment.

Abdominal tuberculosis is a great mimicker. It can mimic a variety of other abdominal conditions/diseases and only a high degree of suspicion can help in the diagnosis otherwise it is likely to be missed or delayed, resulting in high morbidity and mortality.

It should be included in the differential diagnosis of pyrexia of unknown origin, unexplained weight loss, and hepatosplenomegaly. Abdominal tuberculosis should be considered in any patient with unexplained and chronic abdominal symptoms and should be thought of whenever a diagnosis of Crohn's disease or gastrointestinal malignancy is being entertained [17].

Advantages of CT

(1) It gives good anatomic and pathological information regarding different aspects of abdominal tuberculosis.

(2) It helps in assessment of both the intestinal and extraintestinal tuberculosis.

(3) It has high sensitivity and specificity for detecting lymphadenopathy and peritonitis

(4) Use of MDCT scanners can generate near isotropic dataset, which can be post-processed and viewed in various ways using MIP, MPR, and 3D Volumerendered techniques.

Limitations of CT

(1) It requires intravenous iodinated contrast.

(2) The radiation dose is high.

(3) It gives no information regarding bowel peristalsis.

The advantages of MRI are

(1) The lack of ionizing radiation which is particularly important since many of these patients are young.

(2) Better soft tissue contrast resolution in depiction of inflammation and higher visibility of contrast enhancement, safer i.v. contrast agent.

(3) Functional and anatomic assessment of bowel loops can be carried out unlike CT which gives only anatomic information.

(4) In patients with raised creatinine, plain MRI gives much more diagnostic information compared to plain CT.

The disadvantages are

(1) Poor spatial resolution as compared to CT

(2) Long acquisition time requiring good patient compliance

(3) Expensive test with lesser availability.

(4) Is less reliable to detect calcifications and calcium.

\section{Conclusion}

Abdominal tuberculosis is a curable infectious disease with high morbidity and mortality if left untreated. Early diagnosis with adequate anti-tuberculous treatment is imperative. Radiological investigations have become mainstay for the diagnosis of abdominal tuberculosis and to differentiate it from other diseases with similar clinical picture. Both CT and MRI can diagnose abdominal tuberculosis with high sensitivity and specificity. Each have their pros and cons. 
CT scores over MRI in being a quicker, cheaper investigation with better spatial resolution, lesser artefacts and one which can simultaneously evaluate chest, abdomen and pelvis in a single examination.

MRI scores over CT in having no radiation hazard, which is particularly important for young patients and due to its superior soft tissue resolution which gives much more information even without contrast as compared to CT. Thus, in patients with contraindication for intravenous contrast, non-contrast MRI is far better than plain CT. Also, CT gives no information regarding functional assessment of the bowel loops which can be assessed by dynamic cine MRI. In future, technological breakthroughs which can make MRI quicker, improve its spatial resolution will be highly desirable and MRI can substitute CT in many cases and reduce radiation hazard.

The investigation of choice in an individual patient depends on multiple factors like cost, availability, patient compliance, clinical acuity, renal function, need for simultaneous chest evaluation and whether functional assessment of bowel is required.

\section{Compliance with Ethics Guidelines}

Conflict of Interest Dr. Ashish S. Basantani and Dr. Tapan C. Patel each declare no potential conflicts of interest.

Human and Animal Rights and Informed Consent This article does not contain any studies with human or animal subjects performed by any of the authors.

\section{References}

Articles of importance have been highlighted as:

- Of importance

1. Park K. Preventive and Social Medicine: Bhanot; 2011.

2. GOI. TB INDIA 2012. RNTCP Status Report. New Delhi: Central TB Division, Ministry Of Health and Family Welfare; 2010.

3. Ihekwaba FN. Abdominal tuberculosis: a study of 881 cases. J R Coll Surg Edinb. 1993;38(5):293-5.

4. Uygur-Bayramicli O, Dabak G, Dabak R. A clinical dilemma: abdominal tuberculosis. World J Gastroenterol. 2003;9(5): 1098-101.

5. WHO. GLOBAL TUBERCULOSIS REPORT. 2012.

6. Ustianowski A, Shaffer R, Collin S, Wilkinson RJ, Davidson RN. Prevalence and associations of vitamin D deficiency in foreignborn persons with tuberculosis in London. J Infect. 2005;50(5): 432-7.

7. Nnoaham KE, Clarke A. Low serum vitamin D levels and tuberculosis: a systematic review and meta-analysis. Int J Epidemiol. 2008;37(1):113-9.

8. Kumar V, Abbas AK, Fausto N, Mitchell RN. Robbins basic pathology. 8th ed. Philadelphia: Elsevier; 2007.

9. Sharma M, Bhatia V. Abdominal tuberculosis. Indian J Med Res. 2004;120:305-15.
10. Sarrami AH, Sharifi M, Ahsan M, Afsharmoghaddam N. Multiple intestinal perforations as a primary manifestation of abdominal tuberculosis in a HIV-infected patient. JSCR. 2010;10:7.

11. Bhansali S. Abdominal tuberculosis. Experiences with 300 cases. Am J Gastroenterol. 1977;67:324-37.

12. Pombo F, DiazCandamio M, Rodriguez E, Pombo S. Pancreatic tuberculosis: CT findings. Abdom Imaging. 1998;23(4):394-7.

13. Leder RA, Low VH. Tuberculosis of the abdomen. Radiol Clin North Am. 1995;33(4):691-705.

14. Cohan RHDN. The retroperitoneum. In: Haaga JR, editor. Computed tomography and magnetic resonance imaging of the whole body. 3rd ed. St. Louis: Mosby; 1994. p. 1292-326.

15. Sood R. Diagnosis of abdominal tuberculosis: role of imaging. J Indian Acad Clin Med. 2001;2(3):169-77.

16. • De Backer AI, Mortele KJ, Deeren D, Vanschoubroeck IJ, De Keulenaer BL. Abdominal tuberculous lymphadenopathy: MRI features. Eur Radiol. 2005;15(10):2104-9. This article describes MRI appearance of abdominal tuberculous lymphadenopathy in detail.

17. Kapoor VK. Abdominal tuberculosis. Postgrad Med J. 1998;74(874):459-67.

18. Abrams JS, Holden WD. Tuberculosis of the gastrointestinal tract. Arch Surg. 1964;89(2):282-93.

19. Hancock D. Hyperplastic tuberculosis of the distal colon. Br J Surg. 1958;46(195):63-8.

20. Bentley G, Webster J. Gastro-intestinal tuberculosis. A 10-year review. Br J Surg. 1967;54(2):90-6.

21. Bockus H. Tuberculosis of the intestines. Gastroenterology. 1964;2:327-9.

22. Suri S, Gupta S, Suri R. Computed tomography in abdominal tuberculosis. Br J Radiol. 1999;72(853):92-8.

23. - De Backer AI, Mortele KJ, De Keulenaer BL, Henckaerts L, Verhaert L. CT and MR imaging of gastrointestinal tuberculosis. JBR-BTR. 2006;89(4):190-4. This is a study of CT and MRI abdominal tuberculosis which compares both modalities in simple manner.

24. Fidler JL, Guimaraes L, Einstein DM. MR imaging of the small bowel. Radiographics. 2009;29(6):1811-25.

25. Aston NO. Abdominal tuberculosis. World J Surg. 1997;21(5):492-9.

26. Jadvar H, Mindelzun RE, Olcott EW, Levitt DB. Still the great mimicker: abdominal tuberculosis. AJR Am J Roentgenol. 1997;168(6): 1455-60

27. Ha HK, Jung JI, Lee MS, Choi BG, Lee MG, Kim YH, et al. CT differentiation of tuberculous peritonitis and peritoneal carcinomatosis. AJR Am J Roentgenol. 1996;167(3):743-8.

28. Yang ZG, Guo YK, Li Y, Min PQ, Yu JQ, Ma ES. Differentiation between tuberculosis and primary tumors in the adrenal gland: evaluation with contrast-enhanced CT. Eur Radiol. 2006;16(9):2031-6.

29. Thoeni RF, Margulis AR, editors. Gastrointestinal tuberculosis. Seminars in roentgenology; 1979.

30. - Yu RS, Zhang SZ, Wu JJ, Li RF. Imaging diagnosis of 12 patients with hepatic tuberculosis. World J Gastroenterol. 2004;10(11):1639-42. This study gives CT and MRI details of hepatic tuberculosis in different forms.

31. Gibson MS, Puckett ML, Shelly ME. Renal tuberculosis. Radiographics. 2004;24(1):251-6.

32. Jain R, Sawhney S, Bhargava D, Berry M. Gallbladder tuberculosis: sonographic appearance. J Clin Ultrasound. 1995;23(5):327-9.

33. Abu-Zidan FM, Zayat I. Gallbladder tuberculosis (case report and review of the literature). Hepatogastroenterology. 1999;46(29): 2804-6.

34. Nayyar V, Ramakrishna B, Mathew G, Williams RR, Khanduri P. Response to antituberculous chemotherapy after splenectomy. J Intern Med. 1993;233(1):81-3. 
35. Franco-Paredes C, Leonard M, Jurado R, Blumberg HM, Smith RM. Tuberculosis of the pancreas: report of two cases and review of the literature. Am J Med Sci. 2002;323(1):54-8.

36. Ray S, Das K, Mridha AR. Pancreatic and peripancreatic nodal tuberculosis in immunocompetent patients: report of three cases. JOP. 2012;13(6):667-70.

37. Gupta RK, Pandey R, Khan EM, Mittal P, Gujral RB, Chhabra DK. Intracranial tuberculomas: MRI signal intensity correlation with histopathology and localised proton spectroscopy. Magn Reson Imaging. 1993;11(3):443-9.

38. Jung YY, Kim JK, Cho KS. Genitourinary tuberculosis: comprehensive cross-sectional imaging. AJR Am J Roentgenol. 2005; 184(1):143-50.

39. Buxi TB, Sud S, Vohra R. CT and MRI in the diagnosis of tuberculosis. Indian J Pediatr. 2002;69(11):965-72.

40. Kapoor R, Ansari MS, Mandhani A, Gulia A. Clinical presentation and diagnostic approach in cases of genitourinary tuberculosis. Indian J Urol. 2008;24(3):401-5.

41. Fan ZM, Zeng QY, Huo JW, Bai L, Liu ZS, Luo LF, et al. Macronodular multi-organs tuberculoma: CT and MR appearances. J Gastroenterol. 1998;33(2):285-8.

42. Pettengell K, Garb M, Houlder A, Becker P, Simjee A. Radionuclide scintigraphy in tuberculous enteritis. Gastrointest Radiol. 1990;15(2):148-50.

43. - Vanhoenacker FM, De Backer AI, Op de BB, Maes M, Van Altena R, Van Beckevoort D, et al. Imaging of gastrointestinal and abdominal tuberculosis. Eur Radiol. 2004;14(Suppl 3):E103-15. This article summarises salient imaging features of abdominal tuberculosis.

44. De Backer AI, Vanhoenacker FM, Mortele KJ, Vanschoubroeck IJ, De Keulenaer BL, Parizel PM. MRI features of focal splenic lesions in patients with disseminated tuberculosis. AJR Am J Roentgenol. 2006;186(4):1097-102.

45. - Zhang XC, Yang ZG, Li Y, Min PQ, Guo YK, Deng YP, et al. Addison's disease due to adrenal tuberculosis: MRI features. Abdom Imaging. 2008;33(6):689-94. This study describes MRI features in adrenal tuberculosis causing Addisons disease.

46. Sharma AK, Agarwal LD, Sharma CS, Sarin YK. Abdominal tuberculosis in children: experience over a decade. Indian Pediatr. 1993;30(9):1149-53.

47. Khan R, Abid S, Jafri W, Abbas Z, Hameed K, Ahmad Z. Diagnostic dilemma of abdominal tuberculosis in non-hiv patients: an ongoing challenge for physicians. World J Gastroenterol. 2006;12(39):6371-5.

48. Sircar S, Taneja VA, Kansra U. Epidemiology and clinical presentation of abdominal tuberculosis: a retrospective study. J Indian Med Assoc. 1996;94(9):342-4.

49. Amarapurkar DN, Patel ND, Amarapurkar AD, Agal S, Baigal R, Gupte P. Tissue polymerase chain reaction in diagnosis of intestinal tuberculosis and Crohn's disease. J Assoc Physicians India. 2004;52:863-7.

50. Rajput MJ, Memon AS, Rani S, Memon AH. Clinicopathological profile and surgical management outcomes in patients suffering from intestinal tuberculosis. JLUMHS. 2005;4:113-8.

51. Skopin MS, Batyrov FA, Kornilova Z. The prevalence of abdominal tuberculosis and the specific features of its detection. Probl Tuberk Bolezn Legk. 2007;1:22-6.

52. Bandyopadhyay S. Tuberculosis in gastroenterology practice: clinical challenges. Med Update. 2011;21.

53. Lingenfelser T, Zak J, Marks I, Steyn E, Halkett J, Price S. Abdominal tuberculosis: still a potentially lethal disease. Am J Gastroenterol. 1993;88:744. 